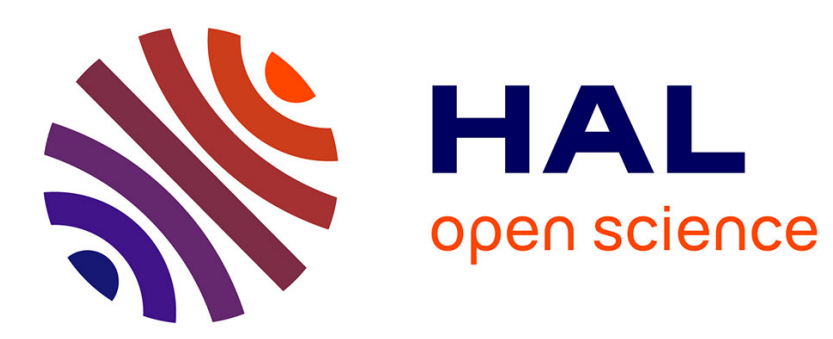

\title{
Le geste efficace: le don du chevalier au saint sur le tympan de Mervilliers (XIIe siècle).
}

\author{
Cécile Voyer
}

\section{To cite this version:}

Cécile Voyer. Le geste efficace: le don du chevalier au saint sur le tympan de Mervilliers (XIIe siècle).. Martin Aurell; Catalina Girbea. Chevalerie \& christianisme aux XIIe et XIIIe siècles., Presses universitaires de Rennes, pp.101-121, 2011, Histoire, 978-2-7535-1726-4. halshs-00682684

\section{HAL Id: halshs-00682684 \\ https://shs.hal.science/halshs-00682684}

Submitted on 25 Sep 2017

HAL is a multi-disciplinary open access archive for the deposit and dissemination of scientific research documents, whether they are published or not. The documents may come from teaching and research institutions in France or abroad, or from public or private research centers.
L'archive ouverte pluridisciplinaire HAL, est destinée au dépôt et à la diffusion de documents scientifiques de niveau recherche, publiés ou non, émanant des établissements d'enseignement et de recherche français ou étrangers, des laboratoires publics ou privés. 


\title{
Le geste efficace: \\ le don du chevalier au saint sur le tympan de Mervilliers ( $\mathrm{XII}^{\mathrm{e}}$ siècle)*
}

\author{
Cécile VOYer
}

L'œuvre sculptée sur le tympan de la porte méridionale de l'église SaintFiacre de Mervilliers a suscité des commentaires ponctuels en raison de son sujet exceptionnel (fig. 1). Elle représente, dans un cadre ecclésial, une scène de don qui associe un miles et un saint. Selon un compartimentage bien pratiqué dans l'art médiéval, deux registres marquent les deux lieux du ciel et de la terre. La zone céleste, lieu de la figuration christique et angélique, offre comme la zone terrestre une certaine symétrie. Toutefois, ces zones se confondent par deux fois: le saint réunit les deux registres, comme l'autel, point de jonction entre le ciel et la terre. La main de Dieu qui apparaît au-dessus de la table sacrificielle sort d'ailleurs des mêmes nuées que l'ange à la gauche du Christ.

Au registre inférieur, le saint en Majesté est entouré par un chevalier et un prêtre, deux des ordines de la société féodale. Le chevalier est associé à son écuyer qui tient sa monture tandis que le prêtre l'est à l'autel surmonté d'un calice. Un motif marginal complète ce registre: un scribe qui tourne le dos à la scène écrit les termes de la donation sur un phylactère qui devient une banderole. L'inscription qui court sur celle-ci devient la bordure de l'image, les mots enserrent donc complètement la représentation de l'offrande. Le texte précise l'identité du chevalier: Rembautus. En revanche, le nom du saint, Georges, est inscrit dans le champ de l'image. Il s'agit vraisemblablement du saint oriental dont les reliques étaient conservées dans la collégiale Saint-Georges de Pithiviers reconstruite au XI ${ }^{\mathrm{e}}$ siècle ${ }^{1}$. La

\footnotetext{
* Nous tenons à remercier vivement Éric Palazzo et Patrick Henriet pour leurs commentaires et leurs observations éclairés, Philippe Durand pour son analyse détaillée de l'équipement du chevalier et Vincent Debiais pour l'étude épigraphique et ses remarques qui ont enrichi considérablement cet article.

1. Miracula ss. Georgii et Lati (BHL 3398d). Ces Miracula ont été écrits par un chanoine de Pithiviers. Le culte de saint Georges était important et bien ancré dans cette région. Il est probable que le saint représenté sur le tympan de Mervilliers soit donc Georges de Lydda dont les reliques étaient conservées dans la collégiale éponyme de Pithiviers.
} 
seigneurie de Pithiviers (Ecclesia sancti Georgii Priverensis) était l'une des plus importantes possessions temporelles de l'évêque d'Orléans.

Le tympan est encastré dans le mur sud de l'ancienne église Saint-Fiacre, aujourd'hui utilisée comme bâtiment agricole. Proche de Toury-en-Beauce, cet édifice dépendait du chapitre de Sainte-Croix d'Orléans et de l'archidiaconé de Beauce ${ }^{2}$. Bien que son histoire soit mal connue, il a pu être dédié à saint Georges avant de passer sous le vocable de Fiacre dont le culte connaît un essor à la fin du XII ${ }^{\text {e }}$ siècle.

Le relief sculpté de Mervilliers a fait l'objet de trois articles entre les dernières décennies du XIXe siècle et les années 1930. Les deux premières études visaient essentiellement la donation et plus particulièrement la nature du don, la transcription de l'inscription, leurs auteurs proposant d'y voir une " charte lapidaire ${ }^{3}$. L'expression est aujourd'hui sujette à caution puisque les inscriptions relatives à des actes privés ou publics n'avaient aucune valeur juridique. Le troisième article était centré sur l'identification du saint à Georges de Lydda et sur la dévotion du miles au saint patron des chevaliers ${ }^{4}$. Reflétant les préoccupations d'une époque qui ne sont plus les nôtres, les auteurs réduisaient l'image à une simple illustration.

Le tympan de Mervilliers a ensuite été brièvement mentionné dans des études iconographiques sur la représentation des laïcs ou des donateurs ${ }^{5}$. Piotr Skubiszewski y fait également référence dans son bel article intitulé "l'intellectuel et l'artiste au Moyen Âge ${ }^{6}$ ". La définition du concepteur d'image étant au cœur de sa réflexion, il s'est intéressé essentiellement à la figure du moine-copiste. Sans poursuivre ses investigations, l'auteur a toutefois relevé le caractère exceptionnel de l'image. Selon lui, la piètre qualité de la réalisation contraste avec la singularité du motif de la banderole sur laquelle court l'inscription rédigée par le scribe: « Nous avons affaire à une scène visiblement "dictée" à un artiste médiocre, artiste qui a su rendre l'essentiel du sujet, mais qui n'a su intégrer sa structure sémantique dans un langage figuratif cohérent. Il est donc impossible d'attribuer le programme

2. Mervilliers: commune de Janville, Marvillare, 1233 (ch. du chap. de Chartres) - Merrvillare, v. 1250 (Pouillé) - Marvilleir, 1264 (Ch. de l'abb. de Josaphat).

3. J. QUicherAT, "Une donation du XII siècle figurée en bas-relief " Revue archéologique, 11, 1854, p. 668-690; M. LECOCQ, "Un symbole d'investiture au Moyen Âge ", Mémoires de la Société archéologique d'Eure-et-Loire, 3, 1863, p. 135-145, notamment p. 144-145; L'abbé SAINSOT, « Le tympan de Mervilliers ", Congrés archéologique de Chartres, 1900, Paris, 1901, p. 96-119.

4. A. LAPeyre, "Le tympan de l'église de Heaulme (Seine-et-Oise) et la légende de saint Georges ", Bulletin Monumental, 45, 1936, p. 317-332.

5. Y. LABANDE-MAILLEFERT, "L'iconographie des laïcs dans la société religieuse aux XI $\mathrm{XI}^{\mathrm{e}}$-XII ${ }^{\mathrm{e}}$ siècles ", I laici nella societas christiana dei secoli $X I^{e}$ e XII $I^{\mathrm{E}}$ Atti della terza seltimana internazionale di studio (21-27 agosto 1965), Milan, 1965, p. 507; A. PRACHE, Ille-de-France romane, La Pierre-qui-vire, 1983, p. 474-475; Cl. MAINES, « Good Works, Social Ties, and the Hope of Salvation: Abbot Suger et Saint-Denis ", Abbot Suger et Saint-Denis, a Symposium, dir. P. Lieber GeSON, The Metropolitan Museum of Art (New York), 1986 p. 82-83.

6. P. SKUBISZEWSKI, «L'intellectuel et l'artiste face à l'œuvre à l'époque romane ", Le travail au Moyen Age, Actes du Colloque international de Louvain-la-Neuve 21-23 mai 1987, Louvain-la-Neuve, 1990, p. 282-283. 
au sculpteur. Il faut voir son auteur dans le moine qui décrit l'événement et qui conçoit en même temps l'image ${ }^{7}$. " Enfin, l'inscription de Mervilliers a été signalée par Robert Favreau dans son article consacré à la notification des actes privés ou publics dans les inscriptions ${ }^{8}$. Si l'auteur y livre une étude magistrale sur la fonction publicitaire de ces inscriptions, celle de Mervilliers n'y est, malheureusement, que partiellement retranscrite. Autrement dit, aucune des précédentes études n'a considéré l'image - la représentation et son inscription - dans sa globalité.

Les chercheurs, notamment André Lapeyre, ont daté la réalisation du tympan autour des années 1120-1140. Selon Yvonne Labande-Mailfert et Anne Prache, la sculpture pourrait avoir été exécutée au début du XII e siècle. Toutefois, une analyse précise de l'équipement de Rembautus invite plutôt à une datation basse dans les dernières décennies du XII ${ }^{\mathrm{e}}$ siècle 9 .

Il convient de préciser avant d'analyser l'image sculptée du tympan de Mervilliers que la qualité de son exécution, jugée médiocre par certains historiens de l'art, est indépendante de sa richesse sémantique.

\section{Les rapports d'autorité et d'ordre}

Saint Georges - identifié par son nom de part et d'autre de sa tête - est assis sur un trône dont la forme semble être un lointain écho du siège curule (fig. 1). Le saint est non seulement vêtu comme un seigneur mais il en a également les gestes. La tête ceinte d'un diadème, le visage encadré par la barbe bien taillée de la "noblesse ", il porte une tunique longue recouverte d'une longue cape retenue par une fibule. En guise de sceptre, il tient vraisemblablement la palme du martyre. Légèrement tourné vers la droite, il s'apprête à recevoir l'offrande que lui tend un miles agenouillé.

Associé au Christ, le saint semble se confondre avec lui. Ses traits sont d'ailleurs similaires à ceux du Dieu Incarné qui est doté d'une identique barbe rase et d'un diadème surmonté d'une croix. Le Christ fait le geste de la parole et tient le Livre dans l'autre main. Les registres terrestres et célestes sont bien séparés: un fin cordon en délimite la frontière, comme les nuées marquent l'appartenance du registre supérieur au céleste. Par sa frontalité, le Christ est détaché du terrestre: son regard évoque l'omnivoyance du principe divin qui englobe le spectateur dans une vision surplombante.

7. Ibid., p. 283.

8. R. FAVREAU, "La notification d'actes publics ou privés par des inscriptions ", dans Cinquante années d'études médiévales. À la confluence de nos disciplines, Actes du colloque organisé à l'occasion du Cinquantenaire du CESCM, Poitiers, $1^{\text {er }} 4$ septembre 2003, Turnhout, 2006, p. 637-664.

9. Nous remercions Philippe Durand qui a réalisé cette étude: le haubert couvrant la totalité du corps n'apparaît qu'après 1150 , les mitaines de mailles et les chausses de mailles vers 1180 , le casque à nasal représenté à Mervilliers disparaît à la fin du XII siècle. L'équipement permet de proposer, grâce à son étude, une datation de la fin du XII ${ }^{\mathrm{e}}$ siècle, peut-être une réalisation au cours des deux dernières décennies de ce siècle. Il convient cependant de remarquer que ce casque est simple pour une période où le heaume commence à apparaître. 
La représentation de la théophanie est ici enrichie par la figuration des anges thuriféraires qui sortent des nuées. Leur encensoir est particulièrement travaillé, détaillé avec soin. La préciosité de l'instrument est associée au geste rituel très prononcé autour du corps du Sauveur. La présence des anges thuriféraires confère non seulement à la représentation christique une dimension liturgique mais elle exalte aussi la dignité de la fonction des officiants.

La verticalité de la représentation du Christ et de Georges est l'expression d'un dispositif hiérarchique, d'un ordre qui s'inscrit sur un axe ciel/ terre. La superposition des têtes, la confusion des corps évoquent la filiation spirituelle entre le Dieu incarné et le saint: Georges est ici le fils, le corps agissant du Père dont il exécute la volonté. Le rapport d'autorité est exprimé par l'association verticale des deux personnages. En liant étroitement la représentation du saint à celle du Christ, le corps christique structure de part en part l'image.

La composition est en effet construite pour signifier les relations d'autorité: si Georges est soumis à l'autorité divine, le jeu des subordinations s'articule horizontalement à partir de son corps. Les enfants du Christ sont disposés de part et d'autre de la figure du saint qui devient à son tour l'expression de l'autorité paternelle, mais aussi, corollaire essentiel de la fonction de père, celle de la protection. Cette organisation horizontale semble en premier lieu signifier l'égalité des fils de Dieu. Le saint procède du Christ selon un axe vertical, suggérant une continuité presque généalogique tandis que l'Ecclésia, réunissant clercs et laïcs, s'ordonne perpendiculairement à cet axe dans l'expression de la germanité.

Cette construction s'appuie, en effet, sur la réflexion théologique de l'équilibre "que le Christ réalise entre ses deux natures divine et humaine ${ }^{10}$ ". Or, la société doit reproduire cet équilibre qui définit les principes de la bonne autorité, fondée elle-même sur deux rapports: le principe hiérarchique et les principes égalitaires - la communauté de nature entre les hommes. Dans les Moralia in Job et dans sa Regula Pastoralis, Grégoire le Grand expose cette tension par « des expressions qui suggèrent un axe horizontal et un axe vertical: extollentia, culmen, prelatio, planities, equalitas ${ }^{11} "$.

Le choix de la composition révèle également la conception duelle - mais non dualiste - de la société (laïcs/clercs), basée sur l'articulation du charnel et du spirituel ${ }^{12}$. L'ordre global de la société, mais aussi de l'univers, repose sur l'équilibre entre ces deux versants de part et d'autre de Dieu. Les corps

10. P. Buc, L'Ambiguïté du livre. Prince, pouvoir et peuple dans les commentaires de la Bible au Moyen Âge, Paris, 1994, p. 46.

11. Ibid., note 68, p. 46.

12. Sur cette question, voir J. BASCHET, La civilisation féodale. De l'an mil à la colonisation de l'Amérique, Paris, 2004, p. 389-425. 
du saint et du Dieu Incarné divisent en effet la composition en deux lieux distincts: à la droite du divin, le siècle et le pouvoir temporel avec son représentant et ses attributs; à sa gauche, le pouvoir spirituel avec son représentant et ses attributs. La composition reflète une certaine symétrie.

Un soin particulier a d'ailleurs été accordé aux détails qui caractérisent chacun des représentants des deux ordres. La fonction chevaleresque est ici précisément définie: le chevalier donateur, coiffé d'un casque à nasal, à bombe arrondie, est revêtu d'un haubert en mailles ${ }^{13}$. Il porte des mitaines, des chausses de mailles et des éperons. Derrière lui, son écuyer attend, en tenant son destrier par la bride. L'épée de taille portée par l'écuyer a été bien mise en valeur. Aux attributs du chevalier répondent ceux du prêtre: la tonsure et l'habit sacerdotal, l'autel et le calice.

Toutefois, la nécessaire articulation du spirituel et du temporel - et la partition réaffirmée de la société - est fondée sur un principe de hiérarchie et de domination du premier sur le second. Dans l'image, ce rapport se traduit par l'agenouillement de Rembautus devant le saint mais aussi devant le prêtre ${ }^{14}$. Associé nettement au martyr trônant, le clerc domine le chevalier. Si le chevalier fait un don à Georges, en pratique, c'est au prêtre qu'il remet son offrande sur l'autel.

\section{Les gestes de la vassalité et le rituel symbolique du don}

Bien que le contexte soit ecclésial, les gestes qui unissent Rembautus à saint Georges évoquent indubitablement les relations féodo-vassaliques. Dans l'attitude du vassal devant son suzerain, le chevalier remet au saint un vase, signe de son engagement. Ses gestes sont les mêmes que ceux de la soumission vassalique: Georges est d'ailleurs figuré trônant dans les atours d'un seigneur. Ici, le chevalier s'en remet au saint comme le vassal à son seigneur. La reproduction des gestes de la vassalité dans le contexte sacré peut sans doute nous éclairer sur le rapport que les bellatores entretenaient avec les puissances célestes. Entre le $\mathrm{XI}^{\mathrm{e}}$ et le XII ${ }^{\mathrm{e}}$ siècle, le chrétien qui prie se comporte face au saint comme un vassal devant son seigneur.

Les gestes "de la deuxième fonction ", celle des guerriers ${ }^{15}$, sont très révélateurs de la manière dont les rapports d'autorité avec les forces célestes sont envisagés par les fidèles et plus particulièrement par les ordines dominants de la société féodale. Ils s'observent d'ailleurs dans une autre

13. L'étude et la datation de l'armement représenté ont été réalisées par Ph. Durand, maître de conférences à l'Université de Bordeaux 3.

14. Voir D. Iogna-Prat, « Ordre(s)», dans Le dictionnaire raisonné de l'Occident médiéval, Paris, 1999, p. $845-860$.

15. Selon J.-Cl. Schmitt, "Les gestes: gestes de la "deuxième fonction " — celle des guerriers, des milites, des bellatores - comme il y a des gestes de la "première fonction " — celle des hommes d'Église ou oratores - et ceux de la "troisième fonction ": les gestes du travail manuel des laboratores. ", La Raison des gestes dans l'Occident médiéval, Paris, 1990, p. 209. 
image, le relief de Théophile de l'abbatiale de Souillac, datée des années 1120-1130 et analysée récemment par Jérôme Baschet ${ }^{16}$ (fig. 2). La confrontation entre Théophile et le Diable y est sculptée à deux reprises: la première montre Théophile lié aux puissances infernales par le pacte dans lequel il renie Dieu (les deux protagonistes ont chacun un doigt sur l'écrit qui les engage); la seconde évoque l'hommage de Théophile au Diable, symbolisé par le rituel de l'immixtio manuum. Le mortel place ses mains dans celles de son nouveau seigneur, le Diable. Or, cette représentation est une interprétation, voire un véritable écart par rapport à la tradition textuelle, des versions de la légende diffusées à partir du IX siècle en Occident ${ }^{17}$. Théophile se livre au diable et lui jure fidélité selon un langage gestuel propre à la vassalité.

À Mervilliers, le miles s'incline humblement devant Georges dans une attitude empruntée aux rituels traditionnels du pouvoir laïque (fig. 3). $\mathrm{Au} \mathrm{VI}^{\mathrm{e}}$ siècle déjà, dans une homélie, Césaire d'Arles appelait de ses vœux l'adoption des mêmes attitudes de déférence pour s'adresser aux puissances temporelles - notamment l'agenouillement et la tête baissée - et à Dieu ${ }^{18}$. Si Dieu est le bénéficiaire de gestes qui traduisent l'attitude de la piété, dans la société des hommes, ce sont les clercs qui en sont destinataires. À la puissance intrinsèque du geste d'échange entre Rembautus et Georges répond l'efficacité symbolique des gestes du prêtre.

Toutefois, la relation qui unit Rembautus au saint renverse en même temps qu'elle reproduit les gestes de l'hommage. Par le biais d'un objet symbolique, le chevalier offre des biens terrestres, quantifiables et passagers, dans l'espoir de recevoir à son tour des biens immatériels, non quantifiables et éternels ${ }^{19}$. Acte rédempteur qui lave le donateur de ses péchés, le don pro anima lui permet alors de " constituer un trésor dans le ciel » comme le suggère l'inscription du bandeau: GAZAS PRESENTES UT HABERET SINE CARENTES. Or l'acte de l'homme n'est qu'un geste de retour après le don inestimable de Dieu qui s'est offert en sacrifice pour sauver l'humanité. Par sa donation, Rembautus s'engage dans une relation avec Dieu qui lui apportera, du moins l'espère-t-il, le Salut.

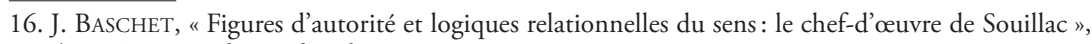
dans L'iconographie médiévale, Paris, 2008, p. 219-225.

17. L'idée de l'hommage vassalique apparaît pour la première fois dans la version de Rutebeuf au XIII ${ }^{\mathrm{e}}$ siècle, Le miracle de Théophile, Paris, 1987. Voir BASCHET, "Figures d'autorité... ", n. 55, p. 414.

18. CÉSAire D’Arles, Sermons au peuple, éd. M.-J Delage, III, Paris, 1986, Sources Chrétiennes 330, Sermons 76-77, p. 229: "Nous essayons d'obtenir un bien terrestre d'un homme fait de terre; et cherchant à obtenir de Dieu la rémission de nos péchés et le repos éternel, nous ne daignons pas baisser la tête ". Voir également sur cette question, SCHMiтT, La Raison des gestes..., p. 58-59.

19. Sur la question du don au Moyen Âge, voir la réflexion historiographique d'E. Magnani, "Les médiévistes et le don. Avant et après la théorie maussienne ", dans Don et Sciences sociales, Théories et pratiques croisées, dir. E. MAGNANI, Dijon, 2007, p. 15-28. 
L'accentuation très importante de la main de Georges insiste sur l'échange qui est en train de s'opérer. Si le saint est l'intermédiaire entre le donateur et le Christ, destinataire de l'offrande, la médiation du prêtre garantit l'efficacité du don. La bénédiction de l'officiant et l'autel témoignent du caractère sacré de la cérémonie.

La représentation de la table sacrificielle est vraisemblablement une allusion au rituel qui consiste à déposer super altare l'objet symbolique du don ${ }^{20}$. Mentionnée dans de nombreuses sources, cette pratique était relativement fréquente pour sceller inexorablement les dons au bénéfice de l'Église comme d'ailleurs ceux de la sphère temporelle ${ }^{21}$. Ainsi, le don dans le cadre des investitures " vassaliques " avait souvent pour cadre le contexte liturgique.

À titre d'exemple, dans l'Introductio monachorum composée au Mont-Saint-Michel, il est spécifié que le duc Richard a déposé par deux fois une charte sur l'autel en faveur de l'abbaye: utriusque igitur, et regis scilicet et domini predicti apostolici, sacrum sape dictus dux Richardus super aram sancti Michaelis ad perenne posuit testimonium, diligenter illud servari pracipiens in posterum ad notitiam sequentium ${ }^{22}$. Il s'agit ici d'un document écrit, mais les objets déposés sur l'autel peuvent être de diverses natures ${ }^{23}$. À Mervilliers, le chevalier tend au saint et en direction de l'autel une sorte d'urne ou de boîte cylindrique. Bien que cet objet ne soit pas répertorié dans l'article de Du Cange sur "L'investiture ", dans certaines chartes émanant de l'aire géographique de production de cette sculpture, il est mentionné un mode d'investiture per thecam, par la boîte ou un reliquaire ${ }^{24}$. Il se peut donc que l'objet symbolique offert à saint Georges soit un réceptacle renfermant un précieux contenu, comme des reliques... Quoi qu'il en soit, l'image de Saint-Fiacre est unique non seulement par son sujet mais aussi par le choix de l'objet symbolique remis par le donateur.

20. Il convient de rappeler que le rituel de l'hommage a pour cadre idéal l'église: «D'ailleurs, il est souvent précisé que ceux des gestes qui le peuvent sont exécutés dans la partie la plus centrale et la plus sacrée de l'édifice, l'autel super altare. C'est sur l'autel que l'on prête serment, c'est sur l'autel qu'est déposé l'objet symbolique de l'investiture ", J. LE GOFF, "Le rituel symbolique de la vassalité ", dans Pour un autre Moyen Âge, Temps, travail et culture en Occident, 18 essais, Paris, 1977 , p. 397.

21. Sur cette question, voir A. ANGENENDT, "Cartam offerre super altare, Zur Liturgisierung von Rechtsvorgängen ", Frühmittelalterliche studien, n 36, 2002, p. 131-158.

22. "Introductio monachorum", dans Chroniques latines du Mont Saint-Michel (IXe-XIte), Les manuscrits du Mont Saint-Michel, Textes fondateurs -1, éd. P. Bouet, O. DesBordes, p. 216-217: IX. 1 Hujus itaque privilegii super altare ipsius sancti Michaelis posuit concessivum donum, sibi retinens tantum potestatem tribuendi fratri electo pastoralem baculum (voilà pourquoi le duc déposa sur l'autel de saint Michel le présent de ce privilège avec cette restriction qu'il ne retenait pour lui que le pouvoir de conférer au frère choisi le bâton pastoral); IX. 3 « Le duc Richard déposa sur l'autel de saint Michel l'acte sacré de ces deux personnages, c'est-à-dire du roi et du pontife apostolique, pour qu'il servît de preuve permanente, ordonnant que ce document fût conservé avec soin, pour que les générations à venir en eussent connaissance ".

23. Du CANGE, "Investitura ", Glossarium medie et infime latinitatis, 3, Paris, éd. 1844, col. 883-892.

24. LeCOCQ, "L'investiture au Moyen Âge... ", p. 144. SAINSOT, "Le tympan de Mervilliers... ", p. 107-109. 
Dans le corpus des images de don, nous en avons choisi une, très riche, qui évoque la pratique de la remise du don sur l'autel par le biais d'un objet symbolique propre au monde chevaleresque ${ }^{25}$. Il s'agit d'une donation du duc de Normandie à l'archange saint Michel $^{26}$ (fig. 4). L'enluminure, réalisée après la seconde moitié du XII ${ }^{\mathrm{e}}$ siècle, figure dans le cartulaire du Mont-Saint-Michel, compilé pour Robert de Thorigni peu après $1154^{27}$. Quatrième et dernier dessin du manuscrit, elle montre sur deux registres la donation de Richard II (996-1026), considéré comme le fondateur de l'abbaye, ou de Robert le Magnifique, au début de son règne, entre 1027 et 1035 . Les quatre images du cartulaire relatent le miracle fondateur de l'abbaye et les principales donations dont elle a bénéficié. Elles offrent ainsi une sorte de parcours visuel de l'histoire de l'abbaye, renforçant la légitimité, voire conférant de la sacralité aux chartes contenues dans le manuscrit. Elles fonctionnent comme l'équivalent de l'attestation visuelle des donations. La première image est inspirée par le récit de l'Apparitio sancti Michaelis in Monte Tumba et montre l'apparition de l'archange à l'évêque d'Avranches pour lui ordonner de lui élever un sanctuaire sur le Mont Tombe. Les trois autres rappellent les plus importantes donations à l'abbaye: la donation de terres au Mont-Saint-Michel par Richard II et la restitution de biens enlevés à l'abbaye par son fils le comte Robert ( $\left.f^{\circ} 19 \mathrm{v}\right)$. Le folio 23v figure la donation de la duchesse Gonor, épouse de Richard I ${ }^{\text {er }}$. Les images sont autant d'éléments dans la construction hagiographique élaborée par la communauté monastique autour de la fondation de l'abbaye et de la constitution de son patrimoine. Marqueurs visuels dans le cartulaire, véritable synthèse des grandes étapes de la fondation de l'abbaye dédiée à l'archange, elles représentent soit une vision, une apparition qui détermine le don, soit les liens de l'abbaye avec les ducs de Normandie. L'image du folio $25 \mathrm{v}$ conjugue ces deux aspects.

Au registre inférieur, encore dans son lit, le duc, couronné, voit apparaitre un ange porteur de la parole divine. Le visionnaire accueille les paroles de l'émissaire céleste. Associé à la scène précédente et pour signifier l'immédiateté de la réponse, le duc, débarrassé des insignes de sa puissance, est agenouillé devant un autel. En présence de deux témoins, il dépose un gant sur l'autel à destination de saint Michel qui lui répond en le désignant.

25. Le rapprochement entre les deux images a déjà été opéré par Maines, "Good works... ", p. 82-83.

26. Sur le cartulaire du Mont-Saint-Michel et ses enluminures (Avranches, BM, ms. 210), voir F. AvRIL, "La décoration des manuscrits au Mont-Saint-Michel (XI $-\mathrm{XII}^{\mathrm{e}}$ siècles) ", Millénaire monastique du Mont Saint-Michel, II, dir. R. ForeviLle, Paris, 1967, p. 207 et 230-232.

27. Avranches, BM, ms. 210, cartulaire du Mont-Saint-Michel, exécuté entre le XII et le XIII ${ }^{\mathrm{e}}$ siècle, fol. 25v. Sur l'enluminure du Mont-Saint-Michel et cette image, M. DosDat, L'enluminure du Mont-Saint-Michel, $X^{e}$-XIt siècles, Rennes, 1991, p. 77. Pour les éditeurs des Chroniques latines du Mont-Saint-Michel..., cette enluminure commémorerait la donation d'Édouard le Confesseur : « le personnage agenouillé prête serment sur le bras droit de saint Aubert dans son bras reliquaire ", p. 242. Nous ne souscrivons pas à cette interprétation. 
L'archange a inspiré le duc qui devient son vassal. Le gant - emblème de l'investiture "vassalique " - symbolise l'action engagée en faveur de saint Michel et de l'abbaye qui lui est vouée. L'édifice sacré a d'ailleurs été représenté avec soin.

Cette scène évoque l'abandon de terres consenti par le duc au profit de l'abbaye ${ }^{28}$. Au registre supérieur, l'acte est réitéré en public mais au profit de l'abbé dans une sorte de transfert de suzeraineté. Pour sa parfaite réalisation, le rituel requiert la présence d'une assistance afin de créer, selon la formule de Jacques Le Goff, « dans l'espace symbolique, un espace social ${ }^{29}$ ". Quatre laïcs, associés au duc, assistent à la scène, garantissant la publicité de l'acte et son caractère officiel. Le donateur, coiffé du capuchon monastique, est agenouillé en prière, dans l'expression de la plus totale humilité. Cette attitude accentue l'idée du renoncement et signifie l'intégration du duc, par le don, à la communauté monastique. Revêtu de ce capuchon, le donateur fait désormais corps avec les membres de l'abbaye et prend place dans les échanges avec l'archange. Le duc se tient d'ailleurs derrière l'abbé, figuré face à l'autel, qui reçoit des mains de saint Michel une verge fleurie. L'archange se dirige vers le guide spirituel de l'abbaye pour lui confier le rameau, symbole de la rétrocession de la donation ducale à l'abbaye. L'épisode se déroule dans l'abbatiale, ce qui rend immuable le transfert de propriété.

Les deux registres du dessin du folio $25 \mathrm{v}$ relatent à travers la donation pro anima la conversion du duc. Ce puissant seigneur devient un humble serviteur de l'archange mais aussi de l'abbé. Sa transformation, de pécheur en homme nouveau, est signifiée par les gestes de prière et par le modeste capuchon monastique qui se sont substitués aux gestes du suzerain remettant sur l'autel son gant et à la couronne ducale. Le duc se défait de l'attitude et des gestes du seigneur, propres au monde féodo-vassalique, figurés au premier registre pour endosser ceux du fidèle, peints au second registre. Le passage d'un état à un autre, propre à la conversion, symbolisé par les deux représentations du duc, est la traduction la plus immédiate de la transformation des biens terrestres en biens célestes. Le puissant seigneur est sur la voie de la Rédemption, son élévation est d'ailleurs évoquée par la composition et la valorisation des gestes du registre supérieur. Il abandonne des biens matériels pour gagner des biens immatériels, garantissant le salut de son âme.

À Mervilliers, le donateur est représenté en sa qualité de chevalier ${ }^{30}$. Non seulement l'inscription qui court autour de la lunette spécifie sa qualité

28. AvriL, «La décoration des manuscrits... », p. 207; DosDAT, L'Enluminure du Mont-SaintMichel..., p. 77

29. Le GofF, « Le rituel symbolique... », p. 398.

30. Si l'épée qui caractérise le chevalier est laissée à la marge de l'espace sacré, Rembautus figure en armes devant saint Georges. 
(miles), mais il est défini visuellement comme un guerrier. Certes ses attributs le qualifient socialement mais il semble que l'on puisse y déceler la valorisation de la fonction guerrière par l'Église. Elle s'inscrit dans une dynamique qui se met en place dès le $\mathrm{XI}^{\mathrm{e}}$ siècle où se forge une éthique chevaleresque religieuse exaltant la fidélité, l'obéissance, la protection des faibles et de l'Église. Par ses dispositions morales et spirituelles, imprimées dans son corps et ses gestes, Rembautus incarne en image l'idéal chevaleresque, soumis à l'Église. La pratique du don, preuve de l'attachement des puissants au modèle de la caritas, témoignait de leur bon comportement social. Ici, la présence du Christ, du saint et du prêtre engage irrémédiablement le donateur.

Le caractère solennel et public de la donation est garanti par l'image et est renforcé par l'inscription ${ }^{31}$. Cette dernière court sur le bandeau du tympan puis, dans l'écoinçon de droite, elle passe sur un phylactère qui retombe sur les genoux d'un moine assis devant une écritoire à deux encriers. Cette banderole sert de support au scribe qui y trace les mots suivants: $[\mathrm{H}]$ ERBER-

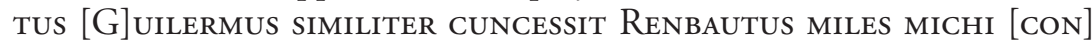
TULIT EJ[US] HERES GAZAS P[RE]SENTES UT SINE CARENTES ${ }^{32}$. En raison de l'absence de ponctuation, la signification de l'inscription est difficile à déterminer. Toutefois, conformément aux formules couramment utilisées dans les chartes, il demeure fort probable que Rembautus ait fait un don pour obtenir son salut en amplifiant celui déjà accordé par ses ancêtres: "Herbertus Guilermus a pareillement concédé, le chevalier Rembautus, son héritier, m’a apporté les trésors présents afin qu'il ait ceux qui n’ont pas de fin".

À Mervilliers, le don dans le contexte liturgique est au cœur de la représentation. Toutefois, certaines images montrent plus explicitement le suffrage des vivants pour le salut des défunts ${ }^{33}$. Aux folios $253^{\mathrm{v}}$ et 254 de l'évangéliaire de Saint-Mihiel, daté de 1040, Irmengarde semble par exemple épauler son époux, Wenher, qui s'apprête à offrir un manuscrit au Christ, lui-même accompagné par l'intercesseur du couple, l'archange Michel ${ }^{34}$. La scène de donation est complétée par un titulus en vers léonins: Laudis amore tua, Michael archangele sancte, Ex Irmengarde, sunt dona parata labore. Tu suscepta deo presenta pro We $[\mathrm{r}]$ nhero, Qui suus ante fuit coniunx, dum corpore vixit, Cuius nunc animam fac perpeti pace beatam. At si quis

31. Sur cette question, voir FAVREAU, "La notification d'actes publics ou privés... », p. 637-664.

32. La transcription de l'inscription et son analyse épigraphique sont dues à Vincent Debiais. En raison de l'absence de la ponctuation, il est impossible d'affirmer précisément qui est le sujet des verbes cuncessit et contulit. S'agit-il de deux actions réalisées par deux sujets différents, Guilermus concède et Rembautus donne ou bien Rembautus concède et apporte?

33. Sur cette question, voir M. LAUWERS, La mémoire des ancêtres, le souci des morts. Morts, rites et société au Moyen Âge (diocèse de Liège, XI'-XIII siècles), Paris, 1996.

34. Sur cette image, voir K. SCHMID, "Zum Stifterbild im Liller Evangelistar des 11. Jarhunders", Frühmittelalterliche Studien, 16, 1982, p. 143-160. 
libro fraudem molitur in isto, Desinat ut cepto, pro Christi nomine posco ${ }^{35}$. Irmengarde offre donc au Christ, par l'intermédiaire de l'archange Michel, un évangéliaire pour garantir le salut de l'âme de son époux défunt. La position dynamique du mari suggère qu'il est au cœur de l'échange entre la donatrice et Dieu. Grâce au don de son épouse, Wenher peut être racheté de ses péchés car il donne par procuration. Irmengarde qui semble littéralement porter son mari soutient d'ailleurs le bras qui tient le codex. Elle présente Wenher au Christ et, grâce au don qu'elle fait au nom de son époux, espère le sauver. En vis-à-vis de la représentation du couple, le Christ tend sa main pour recevoir le codex tandis que saint Michel, l'intercesseur, assiste à la scène.

L'image de Mervilliers commémore à des fins publicitaires la foi pratiquée, l'aumône et le don, expression de la religiosité chevaleresque et du bon comportement. La donation s'est déroulée - les temps de l'inscription sont au passé -, la représentation en fait mémoire, tandis que le scribe, dissocié de la scène représentée par sa position - il lui tourne le dos entérine l'acte par l'écrit (fig. 5). Les mots fixent définitivement les gestes vus et reconnus. Autrement dit, le tympan de Mervilliers offre une définition en image de la valeur efficace de l'acte diplomatique ${ }^{36}$.

\section{Le montage liturgique et la perspective eschatologique}

Comme nous l'avons déjà précisé, le cadre de la donation est clairement signifié. Toutefois, le concepteur de l'image a également insisté sur le contexte liturgique dans lequel se déroule le don du chevalier: l'autel drapé avec le calice ${ }^{37}$, l'officiant vêtu d'une aube, d'une étole à frange, de la chasuble et portant le manipule sur le bras gauche, la cathèdre de Georges, les encensoirs, le livre ouvert tenu par le Christ à la manière des diacres (fig. 1).

La division de l'image en deux registres donne à voir le lien entre la liturgie céleste et les rites dans le cadre ecclésial. Le balancement très marqué des encensoirs des anges ${ }^{38}$ autour de la figure christique fait, en effet, écho à la liturgie terrestre et aux fumées odorantes qui baignent les oblats. La chirophanie, qui surplombe l'autel et son calice, évoque la présence de Dieu au coeur de son église terrestre au moment du sacrifice. Ainsi, la réalisation $\mathrm{du}$ rituel eucharistique ou plus exactement du " complexe eucharistique ",

35. Lille, Université Catholique, ms. 1, Évangéliaire dit de Saint-Mihiel, fol. 253는.254. Si le texte est daté de 1040, les enluminures sont plus tardives.

36. Nous devons à Vincent Debiais cette formule explicite.

37. N. Duval, "L'autel paléochrétien: les progrès depuis le livre de Braun (1924) et les questions à résoudre ", Hortus Artium medievalium, 11, 2005, p. 7-17.

38. Sur l'origine de la représentation des anges thuriféraires, voir M. ANGHEBEN, "Théophanies absidales et liturgie eucharistique. L'exemple des peintures romanes de Catalogne et du nord des Pyrénées comportant un séraphin et un chérubin ", Les Fonts de la pintura romanica, "Ars picta. Temes 1 ", Barcelone, 2008, p. 76-84. 
selon l'expression d'Henri de Lubac ${ }^{39}$, est signifiée à la fois par la présence de la main de Dieu au-dessus de l'autel et par celle du Christ entouré des anges thuriféraires. Avec une économie de moyens indéniable, le concepteur de l'image a réussi à évoquer l'efficacité du rituel et la présence du Christ lors de celui-ci, le sacrifice et le sacrement, autrement dit le signe sensible et la réalité profonde. Le concepteur de la sculpture a pensé en image la liturgie eucharistique et la transformation sacramentelle qui met l'homme visuellement et réellement en présence du corps du Christ.

En figurant le calice sur l'autel, la main de Dieu et le corps christique accompagné des anges thuriféraires, le concepteur du tympan nous offre une définition de l'Eucharistie, à la fois commémoration et réitération, assimilée au corps mystique, au verum corpus.

Le corps eucharistique est ici le corps glorieux du Christ ressuscité, le Sauveur dont la Passion est rappelée par la présence du martyr et par le récipient sacrificiel sur l'autel ${ }^{40}$. Le calice est l'expression même de ce que le Christ s'offre à la messe comme il a fait don de lui-même sur la Croix. Ainsi, le corps eucharistique déposé sur l'autel est le corps glorieux - figuré dans la partie supérieure de l'image - mais également le corps souffrant du Sauveur dans la réitération non sanglante de sa Passion. Dans cette perspective, l'autel, assimilé au Calvaire, est indissociable de la représentation du prêtre qui « manie et brise par les sens - sensualiter - le corps du Christ ${ }^{41}$ ». Selon l'expression d'Alain Rauwel, « l'autel est le lieu où l'on fait le corps du Christ, où l'on fait Dieu (conficere est le verbe le plus couramment employé par les traités d'herméneutique sacrée pour désigner cette production du divin ${ }^{42}$ )». L'autel est également assimilé au corps du Christ ${ }^{43}$. Ce dernier est donc signifié sous différentes formes dans l'image: le calice, l'autel, la main de Dieu, la Maiestas Domini.

L'autel est non seulement le lieu où l'on dépose les saintes espèces, le verum corpus, mais également celui où l'on abrite les reliques des saints ${ }^{44}$. Autrement dit, la table du sacrifice est à la fois le point de jonction entre le ciel et la terre, le trône sur lequel le Christ prend place lors de la consé-

39. H. de Lubac, Corpus Mysticum, L'eucharistie et l'Église au Moyen Âge, Étude historique, Paris, 1944 , p. 77.

40. Sur la question du corps mystique et ses rapports à l'autel, voir A. RAUWEL, "Théologie eucharistique et valorisation de l'autel à l'âge roman ", Hortus Artium medievalium, 11, 2005, p. 177-181.

41. Lanfranc, De Corpore et sangine Domini, PL., 150, col. 410-411.

42. RAUWEL, "Théologie... ", p. 177.

43. PASCHASE RADBERT: "Christus altare creditur esse, hostia et sacrificium, pontifex et sacerdos", Commentaires sur les lamentations de Jérémie, PL., 120, col. 1118.

44. Rauwel rappelle que «l'on n'a peut-être pas assez remarqué que l'Ordo romanus XLII (daté de la première moitié du VIII siècle) prévoit l'introduction dans le sépulcre, avant sa solennelle fermeture, de trois fragments d'hostie, qui viennent se mêler aux reliques des saints ", "Théologie eucharistique... ", p. 178. Sur cette question, voir également, G. J. C. SNOEK, Medieval Piety from Relics to Eucharist: a process of Mutual Interaction, Leyde, 1995. 
cration des Espèces, son tombeau ${ }^{45}$, le Golgotha et le lieu où le corps des saints et celui du Christ se fondent ${ }^{46}$. Selon Ambroise de Milan, les martyrs qui se sont offerts en victimes prennent place sous le Christ, matérialisé par l'autel ${ }^{47}$. Ainsi, lors du rite eucharistique, le corps du saint ou ses reliques étaient concrètement liés au Sauveur. Le concepteur du tympan de Mervilliers a littéralement mis en image les rapports complexes qui unissent les martyrs au Christ. La représentation du Sauveur fait littéralement corps avec celle de Georges à l'instar de l'hostie et de la relique sur et dans l'autel. Autrement dit, la chirophanie au-dessus de l'autel et du calice redouble l'image principale du tympan.

Le saint est ici présenté comme le prolongement du Fils sur terre: ses restes corporels garantissant sa virtus auprès des fidèles. Par l'intermédiaire des fragments de son corps, il est un trait d'union entre le divin et l'humain. Toutefois, c'est bien la Majesté du Christ qui se reflète dans la Majesté du saint, siégeant par procuration sur le trône de gloire du Sauveur.

Or, ce motif est très rare dans les images occidentales en deux dimensions: il est en effet le propre des statues-reliquaires. Sur le tympan de l'abbatiale de Conques, sainte Foy n'est pas représentée en Majesté: elle a quitté son siège d'apparat, évocation de la statue-reliquaire qui était placée derrière l'autel majeur dans le chevet, pour se prosterner devant la main de Dieu $^{48}$ (fig. 6). Le concepteur de l'image a pris soin de préciser le lieu où se déroule la scène. Il s’agit de l'abbatiale de Conques, figurée par les arcades, l'autel et les ex-voto - les entraves des prisonniers, injustement emprisonnés et délivrés par Foy. Le détail de la cathèdre, allusion explicite au trône de la statue-reliquaire, permet, selon l'analyse de Jean-Claude Schmitt, grâce à "un processus de condensation qui s'apparente à celui du rêve ", de suggérer «à la fois l'image visible de la Majesté et l'image onirique de

45. Voir J. Michaud, «Culte des reliques et épigraphie. L’exemple des dédicaces et des consécrations d'autels ", Les reliques, Objets, cultes, symboles, Actes du colloque international de l'université du littoralCôte d'Opale, dir. E. BozoKY et A.-M. HeLvÉTIUs, Turnhout, 1999, p. 199.

46. À partir de la paix constantinienne, une réflexion qui associait reliques et autel, sacrifice du Christ et des martyrs, s'est-elle doucement élaborée, en s'appuyant notamment sur une phrase de l'Apocalypse VI, 9: «Lorsqu'il ouvrit le cinquième sceau, j'aperçus sous l'autel les âmes de ceux qui furent égorgés pour la Parole de Dieu et le témoignage qưils avaient rendu ». Dès 235, Origène met en relation le sacrifice des martyrs et du Christ grâce à l'Apocalypse. Selon lui, les martyrs sont placés sous l'autel céleste, où est offerte la victime unique. Ainsi, cette place privilégiée illustre leur récompense et en même temps augmente le prestige de leur sacrifice. Cette argumentation avait pour but d'expliquer l'intercession des saints placés sous l'autel de l'unique médiateur (ORIGÈNE, Exh. Mart., 30, P.G., 11, col. 601). À ce propos, voir Ch. PIETRI, «L'évolution du culte des saints aux premiers siècles chrétiens ", Les fonctions des saints dans le monde occidental, III'-XIII siècles, Actes du colloque organisé par l'École française de Rome avec le concours de La Sapienza, Rome, 27-29 octobre 1988, Mélanges de l'Ecole française de Rome, 49, 1991, p. 15-36.

47. Ambroise de Milan, Epistole, 22, 12, (CSEL, Ep., 77).

48. Sur le tympan de Sainte-Foy de Conques, voir J.-Cl. Bonne, L'Art roman de face et de profil, Le tympan de Conques, Paris, 1984, et plus particulièrement p. 250; pour une synthèse, nous nous permettons de renvoyer à C. VOYER, Faire le ciel sur la terre, Les images hagiographiques et le décor peint de Saint-Eutrope aux Salles-Lavanguyon (XI' siècle), Turnhout, 2007, p. 350-359. 
la femme ${ }^{49}$ ». À notre connaissance, l'image de Mervilliers constitue donc un unicum. André Grabar qui a mené une magistrale étude sur la représentation du saint en majesté a relevé dans plusieurs textes la formule " trône des martyrs ${ }^{50}$ ». Outre l'image des sièges d'apparat associés au trône de gloire sur lequel prendra place le Christ à la fin des temps (Matth. 19, 28), à la période carolingienne, les chevets des églises ont parfois été désignés par cette expression, comme à Centula par exemple où le fond du chevet oriental était appelé " trône de saint Riquier " car la châsse du saint y était déposée $^{51}$. Or, seul un siècle sépare la statue-reliquaire de Foy de l'assimilation du chevet de l'église-reliquaire/funéraire au trône du saint.

La Majesté du saint sert bien sûr à le glorifier et à marquer son élection, mais le siège d'apparat fait peut-être aussi référence au trône funéraire. Il est, en effet, possible que la mise en scène du trône, auprès du tombeau et à proximité de l'autel, provienne d'un rite mortuaire antique où les membres de la famille du défunt le conviaient à présider le repas commémoratif ${ }^{52}$. En raison de sa passion, le saint était donc tout désigné pour siéger lors des agapes mystiques de la messe. Son sacrifice réitérant celui du Sauveur, le martyr lui était ainsi associé visuellement - par sa cathèdre - et réellement - par ses reliques - lors du rite eucharistique accompli quotidiennement. Si la palme tenue par saint Georges évoque son sacrifice, elle rappelle que le martyr est un imitateur du Fils, que sa passion est un reflet de celle du Christ, un témoignage de sa victoire sur la mort. C'est pourquoi la figure du Sauveur et celle du saint se confondent, comme d'ailleurs l'hostie et les reliques dans l'autel afin de former un pont entre le ciel et la terre. Métaphoriquement, Georges est présenté littéralement comme le Temple de Dieu. C'est à ce titre uniquement que ses restes corporels peuvent être vénérés, la légitimité de leur culte résidant "dans la corporéité et l'historicité du Christ ${ }^{53}$ ", une historicité pensée et mise en scène dans le rite eucharistique.

49. J.-Cl. SCHмiтT, «La légitimation de nouvelles images autour de l'an mil », dans Le corps des images, Essais sur la culture visuelle au Moyen Âge, Paris, 2002, p. 190-192.

50. A. Grabar, "Le trône des martyrs ", Cahiers archéologiques, fin de l'Antiquité et Moyen Âge, 6, 1952 p. 31-41.

51. Selon Grabar « Dans le nord de la France, le chœur de la célèbre abbatiale carolingienne de SaintRiquier ou Centula, près d'Abbeville, était appelé Thronus sanctii Richarii (Plusieurs textes l'attestent comme la Vita s. Angilberti autore Ansihero, chap. VII, von schlosser, Schruftquellen zur Gesch. Der karol. Kunst, p. 262, n 790. Voir aussi Hariulf, Chronique de l'abbaye de Saint-Riquier, éd. F. LOT, p. 297, 298.), "Le trône des martyrs... ", p. 38.

52. Ibid., p. 40.

53. J.-Cl. SchmitT, "Les reliques et les images ", dans Les reliques, Objets, cultes, symboles..., p. 149. Il est intéressant de constater après Bruno Junic que les restes mortels d'un saint conduisent à admettre " le fait que la grâce divine, qui s'est incarnée dans le Christ, agit dans la matière ellemême, dans la chair humaine elle-même, dans l'ensemble des perceptions sensibles de l'humanité ", "La clé d'or et l'idole sanglante. Le culte des reliques entre histoire et anthropologie », dans Liber largitorius, Études d'histoire médiévale offertes à Pierre Toubert par ses élèves, dir. D. BARTHÉLEMY et J.-M. Martin, Genève, 2003, p. 604. 
La simplicité apparente de cette représentation cache une profonde richesse sémantique. Le concepteur de l'image a joué sur plusieurs niveaux selon une dialectique liturgie terrestre-liturgie céleste, corps du Christ-corps du saint, hostie-reliques.

Tout le corps et le sang du Christ sont "réellement " présents dans l'hostie et le calice. Or, le corps eucharistique est ici, comme nous l'avons déjà souligné, le corps glorieux du Christ visible dans la partie supérieure de l'image, encensé par les anges à l'instar des oblats après l'offertoire. Cette représentation donne donc corps aux paroles du prêtre, vicaire du Christ ${ }^{54}$, qui, se substituant au Verbe, déclare que le corps sacramentel est un corps vivant. Ainsi, il nous est offert de contempler à la fois le signe et la réalité.

Il semble également que la composition en deux registres puisse signifier deux angles de vue et deux niveaux de spiritualité différenciés. La théophanie, figurée dans la partie supérieure de l'image, est associée au moment précis du rituel de l'eucharistie. Les anges adorent la Majesté de Dieu dans les cieux conformément à la vision d'Isaïe $(6,1-6)$ dont fait écho la seconde partie du texte de la préface Vere dignum ${ }^{55}$ (Fig. 7). Or, cette vision de face est précisément ce qui se joue au-dessus de l'autel dans le sanctuaire. Comme le souligne Éric Palazzo, «la Maiestas Domini implique de voir le Seigneur dans sa gloire, dans une contemplation face à face avec le spectateur ${ }^{56} "$.

Or, l'espoir du chevalier donateur est de pouvoir contempler Dieu à la fin des Temps. La représentation du Christ est à la fois l'expression de la réalité de la transformation sacramentelle mais peut-être aussi la vision - accessible par les yeux du cœur - pleine de promesse à laquelle aspire Rembautus après le Jugement dernier.

Le chevalier fait un don pour honorer celui inestimable du Christ qui s'est offert en sacrifice pour son salut. En le présentant à l'officiant pour l'Eucharistie, Rembautus rend hommage à ce sacrifice. Débiteur éternel, il répond au geste premier du Fils pour s'engager dans la voie de la Rédemption qu'il appelle de ses vœux. Toute la complexité du don médiéval est signifiée dans cette image. Le contre-don du chevalier s'inscrit dans l'attente eschatologique, dans l'espoir d'une récompense après le Jugement.

Or, la croix pattée du diadème christique interrompt le champ de l'inscription pour marquer le nom du chevalier. De plus, les deux doigts du Christ qui désignent nommément le donateur renforcent l'idée d'une

54. Le livre tenu par le Christ renvoie indubitablement à la fonction sacerdotale, faisant du prêtre son vicaire.

55. Voir sur cette question P. SKUBISZEWSKI, "Maiestas Domini et liturgie », Cinquante années d'études médiévales. À la confluence de nos disciplines, Actes du colloque organisé à l'occasion du cinquantenaire du CESCM, Poitiers, $1^{\mathrm{er}}-4$ septembre 2003, Turnhout, 2005, p. 335-336; AngheBEN, "Théophanies absidales et liturgie eucharistique..." ", p. 76-84.

56. É. PALAzZo, « Le Visible, l'Invisible et les cinq sens dans le haut Moyen Âge, À propos de l'iconographie de l'ivoire de Francfort ", Matérialités et immatérialité dans l'Église au Moyen Âge, Actes du colloque international des 22-23 octobre 2010, Bucarest, 2011, à paraître. 
rédemption accordée à Rembautus ${ }^{57}$. Ainsi, ces détails redoublent les mots qui courent sur le bandeau, créant une équivalence entre les signes iconiques et les signes verbaux: GAZAS PRESENTES UT HABERET SINE CARENTES. Rembautus partagera le destin de ceux qui goûteront aux trésors éternels.

L'inscription est une référence explicite à l'évangile de Luc $(16,9)$ : le prêtre intercède pour le donateur qui, "lorsque les richesses viendront à manquer ", sera reçu par les pauvres et les saints «dans les tabernacles éternels ${ }^{58}$ ". Ainsi, le don pro anima, déposé sur l'autel, est un hommage rendu au Sacrifice christique. Reproduisant la caritas divine, il lave du péché le chevalier qui est alors intégré à la communauté rituelle et peut " prendre place dans les échanges avec Dieu » dont il est l'éternel débiteur.

En faisant un don dans un contexte eucharistique, Rembautus espère que les biens matériels offerts à Dieu par la médiation de l'Église se transforment en biens immatériels et éternels ${ }^{59}$. Autrement dit, il souhaite, conformément à l'eschatocole, que son présent connaisse la même transformation que celle opérée sur l'autel lors de " commutation eucharistique ". Le prêtre est donc un agent indispensable à l'efficacité de la donation: sacrificateur par qui s'opère la " commutation " eucharistique, à travers le service de l'autel, il rend possible la conversion des biens terrestres en biens spirituels ${ }^{60}$.

L'action du scribe est également primordiale: il est en train d'écrire et sa plume plie sous le poids de sa main ${ }^{61}$. Les lettres qu'il trace ou du moins qu'il désigne sont celles du $u t$, conjonction de subordination, qui commande le subjonctif du verbe habeo. En d'autres termes, par ce choix, le concepteur de l'image insiste sur la finalité, sur le but recherché qu’implique le mode choisi : la transformation des biens matériels en trésors éternels. Si l'inscription reprend les formules habituelles des actes privés, la conjonction $u t$, mise en valeur, renvoie au rôle primordial de l'écrit juridique dans la conversion des biens terrestres en biens célestes.

Cette inscription, qui n'a pourtant aucune valeur probatoire, exprime la validité, réelle et efficace, de l'acte écrit ${ }^{62}$ dont l'image est la transposition visuelle. Ainsi, l'image - le rituel représenté - et son inscription - l'écriture - mettent l'accent sur deux sacralités différentes, l'une liturgique et

57. Je remercie Vincent Debiais qui a attiré mon attention sur ces détails cruciaux.

58. En épigraphie funéraire, gazas désigne les récompenses éternelles. Voir à ce sujet l'épitaphe d'Orderic Vital pour la reine Mathilde: gazis dispersis pauper sibi dives egenis/sic infinitate petiit consortia vitel in prima mensis post primam luce novembris (pauvre envers elle-même, par la disposition de ses trésors riche envers les miséreux/elle gagna ainsi les royaumes de la vie éternelle/le lendemain du premier jour du mois de novembre) CIFM 22, 16, p. 52-53. La référence est également Luc 16, 9.

59. Voir Angenendt, "Cartam offere super altare... ", p. 133-158; E. Magnani SoARES-Christen, "Le don au Moyen Âge: pratique sociale et représentations. Perspectives de recherche ", Revue du MAUSS, $1 / 2002, \mathrm{n}^{\circ} 19$, p. 309-322, notamment p. 314-316.

60. Magnani Soares-Christen, "Le don au Moyen Âge... ", p. 317-319.

61. Je remercie Vincent Debiais pour cette remarque. Le paragraphe qui suit est le fruit de ses réflexions.

62. L'emploi des temps au passé, ici le verbe concedere est fréquent dans les actes privés. 
l'autre juridique, qui, ainsi combinées, permettent la transformation des biens matériels en biens immatériels. Tout comme les signes confèrent à l'inscription une validité juridique qu'elle ne possède pas, les rimes sur les mots miles/heres et presentes/carentes donnent l'illusion d'une construction rythmique et métrique de la phrase, en insistant sur ses termes les plus signifiants.

$\mathrm{Si}$, selon l'expression de Robert Favreau, l'inscription et, dans le cas présent, l'image ont une fonction de "publicité durable", elles sont surtout considérées comme efficaces pour accéder aux événements espérés. À Mervilliers, le rapport entre l'écriture et l'image est garant d'efficacité ${ }^{63}$. Lacte du donateur est en effet commémoré mais aussi réitéré par le relief sculpté. Le geste du chevalier est réactualisé à chaque fois que l'image est contemplée. Il est donc fait mémoire de l'offrande qui engage le donateur sur le chemin du Salut. L'image et son inscription figurent un passé réactualisé tout en évoquant le devenir. Ainsi, elles matérialisent la prière perpétuelle de Rembautus qui, à travers elle et le don qu'il a déposé sur l'autel, s'adresse à Dieu ${ }^{64}$.

63. Selon Vincent Debiais, l'inscription est en relief et non gravée: elle est donc, comme l'image, au premier plan, créant une réelle équivalence formelle entre les signes graphiques et les signes iconiques.

64. Sur cette question, voir T. KAMBOUROVA, « Le don et l'image du souverain à Byzance ou de l'apport du don en anthropologie historique des images ", Don et Sciences sociales, Théories et pratiques croisées dir. E. Magnani, Dijon, 2007, p. 87-88. 


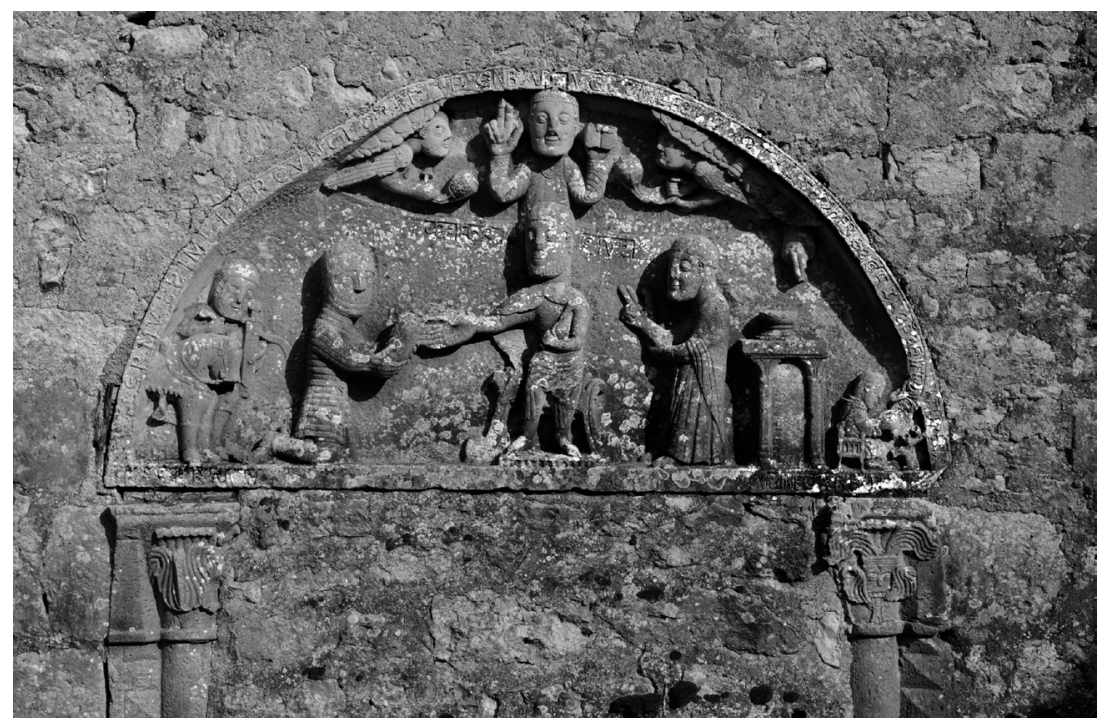

FIG. 1 - Mervilliers, église Saint-Fiacre, tympan, vue d'ensemble (cl. J.-P. BROUARD, CESCM/ CIFM).

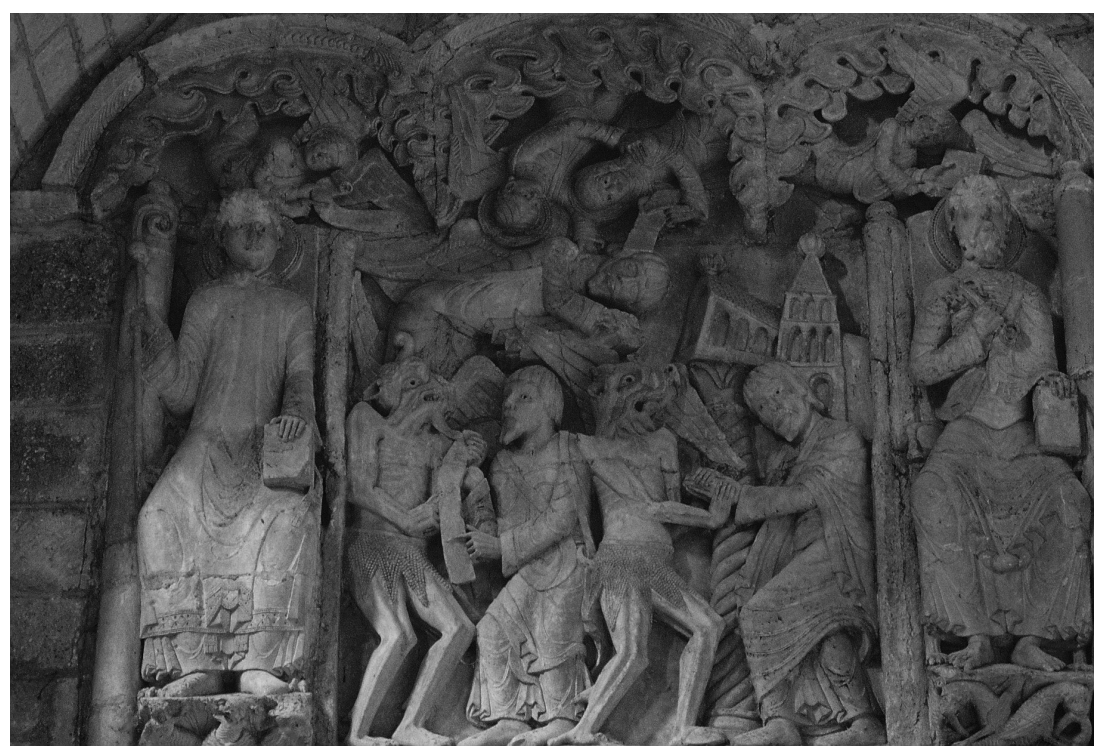

FIG. 2 - Souillac, église Sainte-Marie, revers de la façade, relief de Théophile, Théophile faisant hommage au diable (cl. CESCM, collection J. BASTIEN/B. LEEONET). 


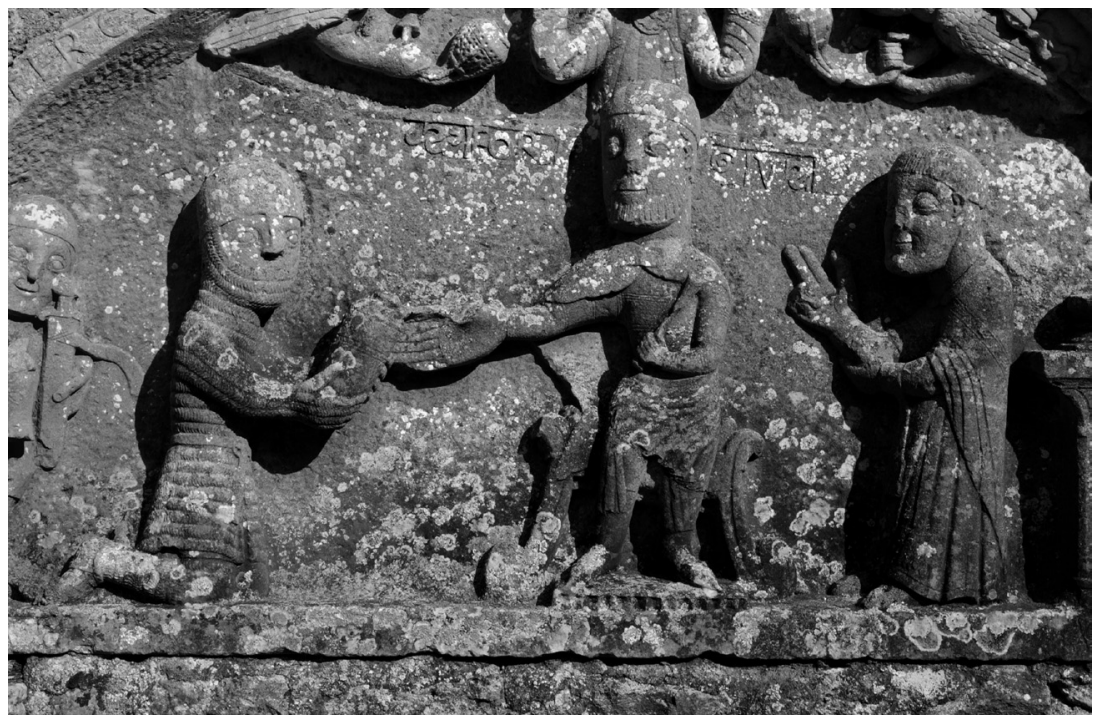

FIG. 3 - Mervilliers, église Saint-Fiacre, tympan, détail (cl. J.-P. BROUARD, CESCM/CIFM).

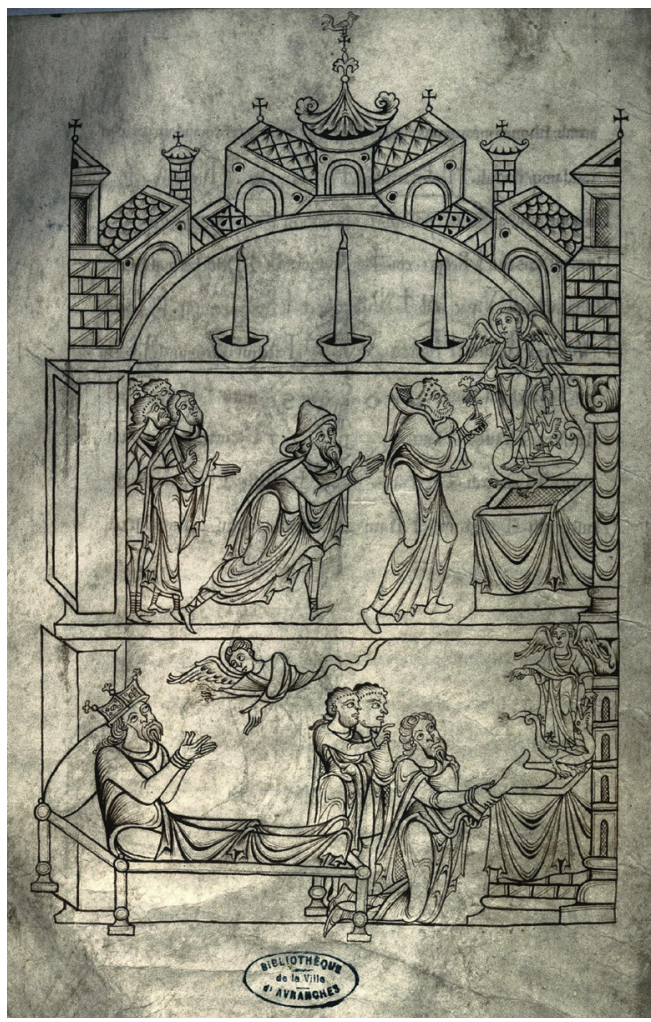

Fig. 4 - Avranches, BM, ms. 210, fo $25 v$, le don du duc de Normandie à saint Michel (cl. IRHT). 
FIg. 5 - Mervilliers, église Saint-Fiacre, tympan, détail (cl. J.-P. BROUARD, CESCM/ CIFM).

FIg. 6 - Conques, église SainteFoy, tympan occidentale, détail (cl. C. VOYER).
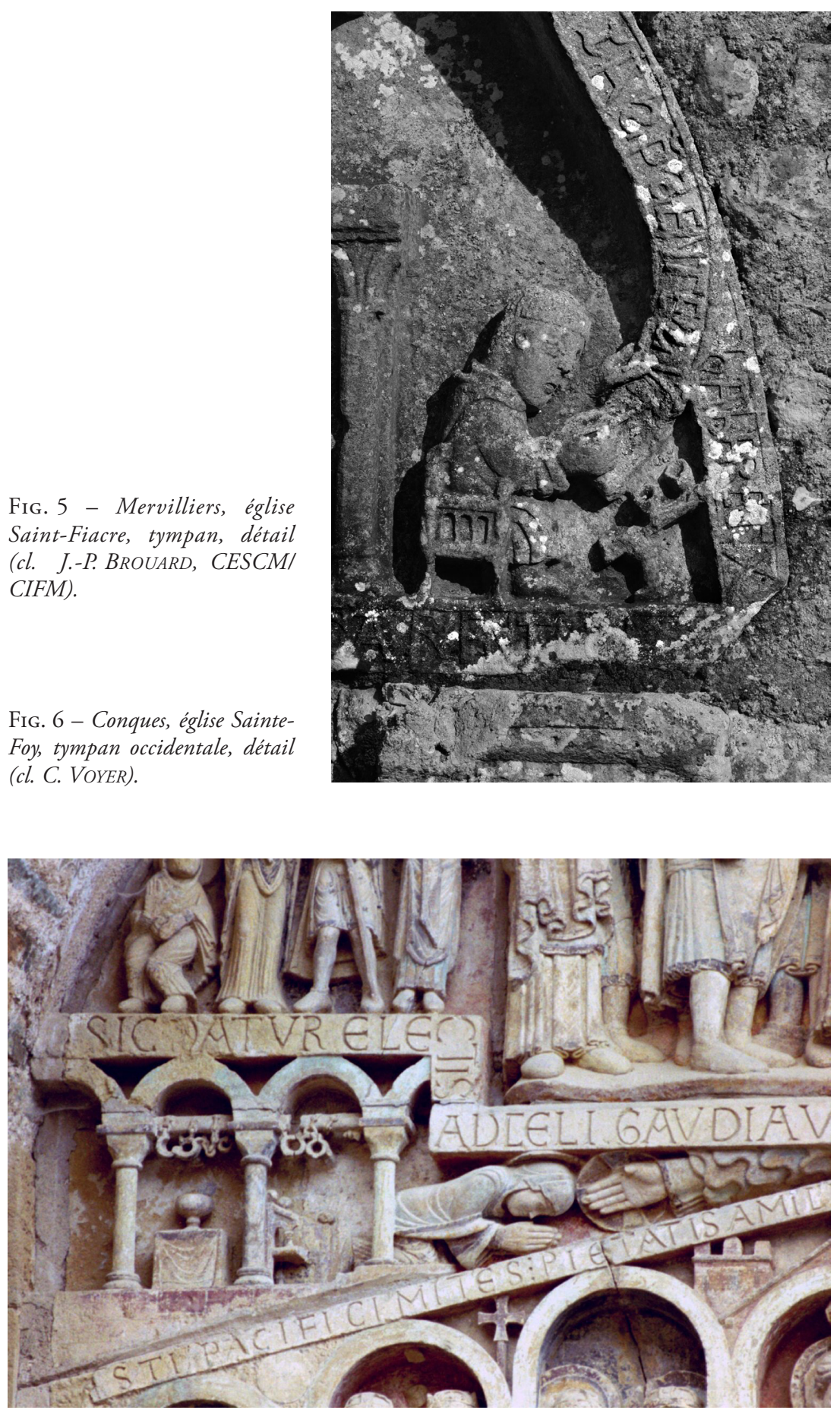


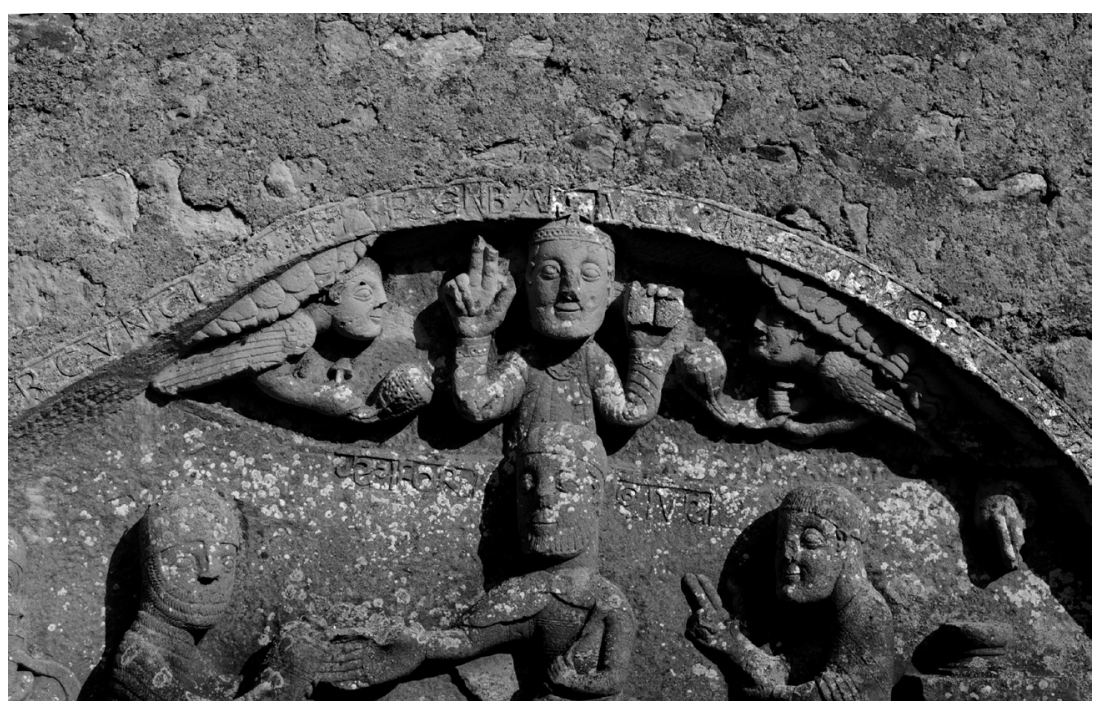

FIG. 7 - Mervilliers, église Saint-Fiacre, tympan, détail (cl. J.-P. BROUARD, CESCM/CIFM). 
